\title{
ANALYZING ON THE DRUG RESISTANCE OF 283 STAPHYLOCOCCUS STRAINS ISOLATED FROM BLOOD CULTURE IN CHILDREN DURING 2009-2010
}

\author{
Q.E. Luo ${ }^{1}$, J.F. $\mathrm{He}^{2}$, S. $\mathrm{Chen}^{1}$ \\ ${ }^{I}$ Ningbo Women's and Children's Hospital, ${ }^{2}$ Pediatrics, Ningbo Women's and Children's Hospital, Ningbo, \\ China
}

Objective: To study the drug resistance rates of 283 staphylococcus strains isolated from blood culture in children during 2009.06-2010.12.

Methods: Blood specimens in medium in bottle were cultured and then rowed on solid medium containing blood. The bacteria were identified with Vitek system and additional analysis on drug resistance were finished with WHONET 5.4 software. $\chi^{2}$ test was used in the study.

Results: Two hundred and eighty-three staphylococcus strains were isolated and the constituent ratio was $58.0 \%$, and $83.4 \%$ of the strains were isolated from neonate. Staphylococcus epidermidis (occupuy $48.4 \%$ ) was the most common strains, followed by staphylococcus simulation (13.4\%) and staphylococcus haemolyticus (12.7\%). Only 5 staphylococcus aureus strains (1.8\%) were identified in the study. Of all 283 strains, $98.8 \%$ were $\beta$ lactamase positive and resistant to penicillin $\mathrm{G}$, and $76.7 \%$ of all strains were resistant to oxacillin. The resisitant rates to erythromycine, sulfamethoxazole, clindamycin, levofloxacin and gentamicin were $86.6 \%, 60.1 \%, 39.6 \%, 38.9 \%$ and $33.9 \%$, respectively. All strains were sensitive to vancomycin and linezolid. The resistant rate to oxacillin of staphylococcus epidermidis was $65.7 \%$, which was significantly higher than that of staphylococcus simulation $\left(97.4 \%, \chi^{2}=13.44, P<0.05\right)$ and staphylococcus haemolyticus $\left(94.4 \%, \chi^{2}=10.23, P<0.05\right)$.

Conclusion: The resistant rates of staphylococcus to oxacillin were high, however, the resistant rate of staphylococcus epidermidis to oxacillin was lower than that in other staphylococcus. It is imperative to choose antibiotics according to result of drug sensitive test. 